\title{
Semi-supervised adversarial neural networks for single-cell classification
}

Jacob C. Kimmel

Calico Life Sciences, LLC

South San Francisco, CA, 94080

jacob@calicolabs.com
David R. Kelley

Calico Life Sciences, LLC

South San Francisco, CA, 94080

drk@calicolabs.com

* Lead Contact 


\begin{abstract}
Annotating cell identities is a common bottleneck in the analysis of single cell genomics experiments. Here, we present scNym, a semi-supervised, adversarial neural network that learns to transfer cell identity annotations from one experiment to another. scNym takes advantage of information in both labeled datasets and new, unlabeled datasets to learn rich representations of cell identity that enable effective annotation transfer. We show that scNym effectively transfers annotations across experiments despite biological and technical differences, achieving performance superior to existing methods. We also show that scNym models can synthesize information from multiple training and target datasets to improve performance. In addition to high accuracy, we show that scNym models are well-calibrated and interpretable with saliency methods.
\end{abstract}

Keywords single cell $\cdot$ neural network $\cdot$ cell type classification $\cdot$ semi-supervised learning $\cdot$ adversarial learning

\title{
Introduction
}

Single cell genomics allows for simultaneous molecular profiling of thousands of diverse cells and has advanced our understanding of development [Trapnell, 2015], aging [Angelidis et al., 2019, Kimmel et al., 2019, Ma et al., 2020], and disease [Tanay and Regev, 2017]. To derive biological insight from these data, each single cell molecular profile must be annotated with a cell identity, such as a cell type or state label. Traditionally, this task has been performed manually by domain expert biologists. Manual annotation is time consuming, somewhat subjective, and error prone. Annotations influence the results of nearly all downstream analyses, motivating more robust algorithmic approaches for cell type annotation.

Automated classification tools have been proposed to transfer annotations across datasets [Kiselev et al., 2018,

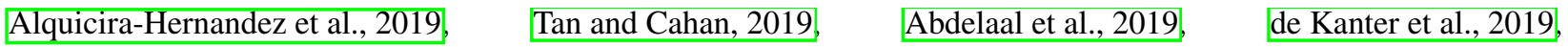
Pliner et al., 2019, Zhang et al., 2019|. These existing tools learn relationships between cell identity and molecular features from a training set with existing labels without considering the unlabeled target dataset in the learning process. However, results from the field of semi-supervised representation learning suggest that incorporating information from the target data during training can improve the performance of prediction models [Kingma et al., 2014, Oliver et al., 2018, Verma et al., 2019, Berthelot et al., 2019]. This approach is especially beneficial when there are systematic differences - a domain shift - between the training and target datasets. Domain shifts are commonly introduced between single cell genomics experiments when cells are profiled in different experimental conditions or using different sequencing technologies.

A growing family of representation learning techniques encourage classification models to provide consistent interpolations between data points as an auxiliary training task to improve performance [Verma et al., 2019, Berthelot et al., 2019]. In the semi-supervised setting, the MixMatch approach implements this idea by "mixing" 
observations and their labels with simple weighted averages. Mixed observations from the training and target datasets form a bridge in feature space, encouraging the model to learn a smooth interpolation across the domains. Another family of techniques seek to improve classification performance in the presence of domain shifts by encouraging the model to learn a representation in which observations from different domains are embedded nearby, rather than occupying distinct regions of a latent space [Wilson and Cook, 2020]. One successful approach uses a "domain adversary" to encourage the classification model to learn a representation that is invariant to dataset-specific features [Ganin et al., 2016]. Both interpolation consistency and domain invariance are desirable in the single cell genomics setting, where domain shifts are common and complex gene expression boundaries separate cell types.

Here, we introduce a cell type classification model that uses semi-supervised and adversarial machine learning techniques to take advantage of both labeled and unlabeled single cell datasets. We demonstrate that this model offers superior performance to existing methods and effectively transfers annotations across different animal ages, perturbation conditions, and sequencing technologies. Additionally, we show that our model learns biologically interpretable representations and offers well-calibrated metrics of annotation confidence that can be used to make new cell type discoveries.

\section{Results}

\section{scNym}

In the typical supervised learning framework, the model touches the target unlabeled dataset to predict labels only after training has concluded. By contrast, our semi-supervised learning framework trains the model parameters on both the labeled and unlabeled data in order to leverage the structure in the target dataset, whose measurements may have been influenced by myriad sources of biological and technical bias and batch effects. While our model uses observed cell profiles from the unlabeled target dataset, at no point does the model access ground truth labels for the target data. Ground truth labels on the target dataset are used exclusively to evaluate model performance. Some single cell classification methods require manual marker gene specification prior to model training. scNym requires no prior manual specification of marker genes, but rather learns relevant gene expression features from the data.

scNym uses the unlabeled target data through a combination of MixMatch semi-supervision [Berthelot et al., 2019] and by training a domain adversary [Ganin et al., 2016] in an iterative learning process (Fig. [1]A, Methods). The MixMatch semi-supervision approach combines MixUp data augmentations [Zhang et al., 2018, Thulasidasan et al., 2019] with pseudolabeling of the target data [Lee, 2013, Verma et al., 2019] to improve generalization across the training and target domains. At each training iteration, we "pseudolabel" unlabeled cells using predictions from the classification model, then augment each cell profile using a biased weighted average of gene expression and labels with another randomly chosen cell (Fig. 1B B). The resulting mixed profiles are dominated by a single cell, adjusted modestly to more closely resemble another. As part of MixMatch, we mix profiles across the training and unlabeled data, so that some of the resulting mixed profiles are interpolations between the two datasets. We fit the model parameters to minimize cell 
type classification error on these mixed profiles, encouraging the model to learn a general representation that allows for interpolation between observed cell states.

The scNym classifier learns a representation of cell identity in the hidden neural network layers where cell types are linearly separable. Alongside, we train an adversarial model to predict the domain-of-origin for each cell (e.g. training set, target set) from this learned embedding. We train the scNym classifier to compete with this adversary, updating the classifier's embedding to make domain prediction more difficult. At each iteration, the adversary's gradients highlight features in the embedding that discriminate the different domains. We update the scNym classifier using the inverse of the adversarial gradients, reducing the amount of domain-specific information in the embedding as training progresses. This adversarial training procedure encourages the classification model to learn a domain-adapted embedding of the training and target datasets that improves classification performance (Fig. (TC). In inference mode, scNym predictions provide a probability distribution across all cell types in the training set for each target cell.

\section{scNym transfers cell annotations across biological conditions}

We evaluated the performance of scNym transferring cell identity annotations in eleven distinct tasks. These tasks were chosen to capture diverse kinds of technological and biological variation that complicate annotation transfer. Each task represents a true cell type transfer across different experiments, in contrast to some efforts that report within-experiment hold-out accuracy.

We first evaluated cell type annotation transfer between animals of different ages. We trained scNym models on cells from young rats (5 months old) from the Rat Aging Cell Atlas [Ma et al., 2020] and predicted on cells from aged rats (27 months old, Fig. 22A, Methods). We found that predictions from our scNym model trained on young cells largely matched the ground truth annotations (92.2\% accurate) on aged cells (Fig. 2B, C).

We compared scNym performance on this task to state of the art single cell identity annotation methods Kiselev et al., 2018, Alquicira-Hernandez et al., 2019, Tan and Cahan, 2019, Abdelaal et al., 2019, de Kanter et al., 2019]. We also compared scNym to state of the art unsupervised data harmonization methods [Korsunsky et al., 2019, Stuart et al., 2019, Xu et al., 2019, Tran et al., 2020] followed by supervised classification with a support vector machine, for a total of ten baseline approaches (Methods). scNym produced significantly improved labels over these methods, some of which could not complete this large task on our hardware (256GB RAM) (Wilcoxon Rank Sums on accuracy or $\kappa$-scores, $p<0.01$, Fig. 2D, Table 11. scNym runtimes were competitive with baseline methods (Fig. S1]. We found that some of the largest differences in accuracy between scNym and the commonly used scmap-cell method were in the skeletal muscle. scNym models accurately classified multiple cell types in the muscle that were confused by scmap-cell (Fig. 2E), demonstrating that the increased accuracy of scNym is meaningful for downstream analyses.

We next tested the ability of scNym to classify cell identities after perturbation. We trained on unstimulated human peripheral blood mononuclear cells (PBMCs) and predicted on PBMCs after stimulation with IFNB1 
(Fig. 3A) [Kang et al., 2017]. scNym achieved high accuracy ( $>91 \%$ ), superior to baseline methods (Fig. 3C, Table 1). The common scmap-cluster method frequently confused monocyte subtypes, while scNym did not (Fig. 33B).

Cross-species annotation transfer is another context where distinct biology creates a domain shift across training and target domains. To evaluate if scNym could transfer labels across species, we trained on mouse cells with either rat or human cells as target data and observed high performance (Fig. S2).

\section{scNym models learn biologically meaningful cell type representations}

To interpret the classification decisions of our scNym models, we developed integrated gradient analysis tools to identify genes that influence model decisions (Methods)[Sundararajan et al., 2017]. The integrated gradient method attributes the prediction of a deep network to its input features, while satisfying desirable axioms of interpretability that simpler methods like raw gradients do not. For the PBMC cross-stimulation task, we found that salient genes included known markers of specific cell types such as $C D 79 A$ for B cells and $G N L Y$ for NK cells. Integrated gradient analysis also revealed specific cell type marker genes that may not have been selected a priori, such as NCOA4 for megakaryocytes (Fig. 3D, E, Fig. S3). We also performed integrated gradient analysis for a cross-technology mouse cell atlas experiment (described below) and found that marker genes chosen using scNym integrated gradients were superior to markers chosen using SVM feature importance scores based on Gene Ontology enrichment (Fig. S4). These results suggest that our models learned biologically meaningful representations that are more generalizable to unseen cell profiles, regardless of condition or technology.

We also used integrated gradient analysis to understand why the scNym model misclassified some FCGR $3 \mathrm{~A}^{+}$monocytes as $\mathrm{CD}_{1} 4^{+}$monocytes in the PBMC cross-stimulation task (Methods). This analysis revealed genes driving these incorrect classifications, including some $\mathrm{CD} 14^{+}$monocyte marker genes that are elevated in a subset of FCGR3A ${ }^{+}$ monocytes (Fig. 3F). Domain experts may use integrated gradient analysis to understand and review model decisions for ambiguous cells.

\section{scNym transfers annotations across single cell sequencing technologies}

To evaluate the ability of scNym to transfer labels across different experimental technologies, we trained on single cell profiles from ten mouse tissues in the Tabula Muris captured using the 10x Chromium technology and predicted labels for cells from the same compendium captured using Smart-seq2 [Tabula Muris Consortium, 2018]. We found that scNym predictions were highly accurate $(>90 \%)$ and superior to baseline methods (Fig. S5 A, B, C). scNym models accurately classified monocyte subtypes, while baseline methods frequently confused these cells (Fig. [S5D, E). In a second cross-technology task, we trained scNym on mouse lung data from the Tabula Muris and predicted on lung data from the Mouse Cell Atlas, a separate experimental effort that used the Microwell-seq technology [Han et al., 2018]. We found that scNym yielded high classification accuracy ( $>90 \%$ ), superior to baseline methods, despite experimental batch effects and differences in the sequencing technologies (Fig. S6). We also trained scNym models to transfer 
regional identity annotations in spatial transcriptomics data and found performance competitive with baseline methods (Fig. [S7]. Together, these results demonstrate that scNym models can effectively transfer cell type annotations across technologies and experimental environments.

\section{Multi-domain training allows integration of multiple reference datasets}

The number of public single cell datasets is increasing rapidly [Svensson et al., 2018]. Integrating information across multiple reference datasets may improve annotation transfer performance on challenging tasks. The domain adversarial training framework in scNym naturally extends to training across multiple reference datasets. We hypothesized that a multi-domain training approach would allow for more general representations that improve annotation transfer. To test this hypothesis, we evaluated the performance of scNym to transfer annotations between single cell and single nucleus RNA-seq experiments in the mouse kidney. These data contained six different single cell preparation methods and three different single nucleus methods, capturing a range of technical variation in nine distinct domains [Denisenko et al., 2020](Fig. 4A, B).

scNym achieved significantly greater accuracy than baseline methods transferring labels from single nucleus to single cell experiments using multi-domain training. This result was also achieved for the inverse transfer task, transferring annotations from single cell to single nucleus experiments (tied with best baseline, Fig. 4C, Table 1). We found that scNym delivered more accurate annotations for multiple cell types in the cell to nucleus transfer task, including mesangial cells and tubule cell types (Fig. 4D, E). These improved annotations highlight that the performance advantages of scNym are meaningful for downstream analysis and biological interpretation. We found that multi-domain scNym models achieved greater accuracy than any single domain model on both tasks and effectively synthesized information from single domain training sets of varying quality (Fig. 4F, Fig. [S8). We performed a similar experiment using data from mouse cortex nuclei profiled with four distinct single cell sequencing methods, training on three methods at a time and predicting annotations for the held-out fourth method for a total of four unique tasks. scNym was the top ranked method across tasks (Fig. S9).

\section{scNym confidence scores enable expert review and allow new cell type discoveries}

Calibrated predictions, in which the classification probability returned by the model precisely reflects the probability it is correct, enable more effective interaction of the human researcher with the model output. We investigated scNym calibration by comparing the prediction confidence scores to prediction accuracy (Methods). We found that semisupervised adversarial training improved model calibration, such that high confidence predictions are more likely to be correct (Fig. 51A, B; Fig. S10A, B; Fig. S11). scNym confidence scores can therefore be used to highlight cells that may benefit from manual review (Fig. S10 C, Fig. $\mathbf{S 1 1 B}$ ), further improving the annotation exercise when it contains a domain expert in the loop.

scNym confidence scores can also highlight new, unseen cell types in the target dataset using an optional pseudolabel thresholding procedure during training, inspired by FixMatch [Sohn et al., 2020] (Methods). The semi-supervised 
and adversarial components of scNym encourage the model to find a matching identity for cells in the target dataset. Pseudolabel thresholding allows scNym to exclude cells with low confidence pseudolabels from the semi-supervised and adversarial components of training, stopping these components from mismatching unseen cell types and resulting in correctly uncertain predictions.

To test this approach, we simulated two experiments where we "discover" multiple cell types by predicting annotations on the Tabula Muris brain cell data using models trained on non-brain tissues (Fig. 5AA, B; Methods). We first used pre-trained scNym models to predict labels for new cell types not present in the original training or target sets, and scNym correctly marked these cells with low confidence scores (Fig. [S12). In the second experiment, we included new cell types in the target set during training and found that scNym models with pseudolabel thresholding correctly provided low confidence scores to new cell types, highlighting these cells as potential cell type discoveries for manual inspection (Fig. 55C, D; Fig. S13.

We found that scNym embeddings capture cell type differences even within the low confidence cell population, such that clustering these cells in the scNym embedding can provide a hypothesis for how many new cell types might be present (Fig. S14). We also found that putative new cell types could be discriminated from other low confidence cells, like prediction errors on a cell type boundary (Fig. S15). These results demonstrate that scNym confidence scores can highlight target cell types that were absent in the training data, potentially enabling new cell type discoveries.

\section{Semi-supervised and adversarial training components improve annotation transfer}

We ablated different components of scNym to determine which features were responsible for high performance. We found that semi-supervision with MixMatch and training with a domain adversary improved model performance across multiple tasks (Fig. 6B, Fig. S16). We hypothesized that scNym models might benefit from domain adaptation through the adversarial model by integrating the cells into a latent space more effectively. Supporting this hypothesis, we found that training and target domains were significantly more mixed in scNym embeddings (Fig. S17). These results suggest that semi-supervision and adversarial training improve the accuracy of cell type classifications.

\section{scNym is robust to hyperparameter selection}

Hyperparameter selection can be an important determinant of classification model performance. In all tasks presented here, we have used the same set of default scNym parameters derived from past recommendations in the representation learning literature (Methods). To determine how sensitive scNym performance is to these hyperparameter choices, we trained scNym models on the hPBMC cross-stimulation task across a grid of hyperparameter values. We found that scNym is robust to hyperparameter changes within an order of magnitude of the default values, demonstrating that our defaults are not "overfit" to the benchmark tasks presented here (Fig. [S18]. We also performed hyperparameter optimization using reverse 5-fold cross-validation for the top three baseline methods (SVM, singleCellNet, scmap-cellexact) to determine if an optimized baseline was superior to scNym across four benchmarking tasks (Methods). We 
found that scNym performance using default parameters was superior to the performance of baseline methods after hyperparameter tuning (Table S3, Fig. S19].

\section{Discussion}

Single cell genomics experiments have become more accessible due to commercial technologies, enabling a rapid increase in the use of these methods [Svensson et al., 2020]. Cell identity annotation is an essential step in the analysis of these experiments, motivating the development of high performance, automated annotation methods that can take advantage of diverse datasets. Here, we introduced a semi-supervised adversarial neural network model that learns to transfer annotations from one experiment to another, taking advantage of information in both labeled training sets and an unlabeled target dataset.

Our benchmark experiments demonstrate that scNym models provide high performance across a range of cell identity classification tasks, including cross-age, cross-perturbation, and cross-technology scenarios. scNym performs better in these varied conditions than ten state of the art baseline methods, including three unsupervised data integration approaches paired with supervised classifiers (Fig. 6A, Table 11). The superiority of scNym is consistent across diverse performance metrics, including accuracy, Cohen's $\kappa$-score, and the multi-class receiver operating characteristic (MCROC; Fig. [S20, Table S1], Table S2].

The key idea that differentiates scNym from previous cell classification approaches is the use of semi-supervised [Berthelot et al., 2019] and adversarial training [Ganin et al., 2016] to extract information from the unlabeled, target experiment we wish to annotate. Through ablation experiments, we showed that these training strategies improve the performance of our models. Performance improvements were most pronounced when there were large, systematic differences between the training and target datasets (Fig. 3). Semi-supervision and adversarial training also allow scNym to integrate information across multiple training and target datasets, improving performance (Fig. 4). As large scale single cell perturbation experiments become more common [Dixit et al., 2016, Srivatsan et al., 2019] and multiple cell atlases are released for common model systems, our method's ability to adapt across distinct biological and technical conditions will only increase in value.

Most downstream biological analyses rely upon cell identity annotations, so it is important that researchers are able to interpret the molecular features that drive model decisions. We showed that backpropagation-based saliency analysis methods are able to recover specific cell type markers, confirming that scNym models learn interpretable, biologically relevant features of cell type. In future work, we hope to extend upon these interpretability methods to infer perturbations that alter cell identity programs using the informative representations learned by scNym. 


\section{Methods}

\section{scNym Model}

Our scNym model $f_{\theta}$ consists of a neural network with an input layer, two hidden layers, each with 256 nodes, and an output layer with a node for each class. The first three layers are paired with batch normalization [Ioffe and Szegedy, 2015], rectified linear unit activation, and dropout [Srivastava et al., 2014]. The final layer is paired with a softmax activation to transform real number outputs of the neural network into a vector of class probabilities. The model maps cell profile vectors $x$ to probability distributions $p(y \mid x)$ over cell identity classes $y$.

$$
p(y \mid x)=f_{\theta}(x)
$$

We train scNym to map cell profiles in a gene expression matrix $x \in \mathbf{X}^{\text {Cells } \times \text { Genes }}$ to paired cell identity annotations $y \in \mathbf{y}$. Transcript counts in the gene expression matrix are normalized to counts per million (CPM) and log-transformed after addition of a pseudocount $(\log (\mathrm{CPM}+1))$. During training, we randomly mask $10 \%$ of genes in each cell with 0 values, then renormalize to obtain an augmented profile.

We use the Adadelta adaptive stochastic gradient descent method [Zeiler, 2012] with an initial learning rate of $\eta=1.0$ to update model parameters on minibatches of cells, with batch sizes of 256 . We apply a weight decay term of $\lambda_{\mathrm{WD}}=10^{-4}$ for regularization. We train scNym models to minimize a standard cross-entropy loss function for supervised training.

$$
L_{\mathrm{CE}}\left(\mathbf{X}, f_{\theta}\right)=\mathbb{E}_{(x, y) \sim(\mathbf{X}, \mathbf{y})}\left[-\sum_{k=1}^{K} y_{(k)} \log \left(f_{\theta}(x)\right)_{k}\right]
$$

where $y_{(k)}$ is an indicator variable for the membership of $x$ in class $k$, and $k \in K$ represent class indicators.

We fit all scNym models for a maximum of 400 epochs and selected the optimal set of weights using early stopping on a validation set consisting of $10 \%$ of the training data. We initiate early stopping after training has completed at least $5 \%$ of the total epochs to avoid premature termination.

Prior to passing each minibatch to the network, we perform dynamic data augmentation with the "MixUp" operation [Zhang et al., 2018]. MixUp computes a weighted average of two samples $x$ and $x^{\prime}$ where the weights $\lambda$ are randomly sampled from a Beta distribution with a symmetric shape parameter $\alpha$.

$$
\operatorname{Mix}_{\lambda}\left(x, x^{\prime}\right)=\lambda x+(1-\lambda) x^{\prime} ; \lambda \sim \operatorname{Beta}(\alpha, \alpha)
$$

For all experiments here, we set $\alpha=0.3$ based on performance in the natural image domain [Zhang et al., 2018]. Forcing models to interpolate predictions smoothly between samples shifts the decision boundary away from highdensity regions of the input distribution, improving generalization. This procedure has been shown to improve classifier 
performance on multiple tasks [Zhang et al., 2018]. Model calibration - the correctness of a model's confidence scores for each class - is generally also improved by this augmentation scheme [Thulasidasan et al., 2019].

\section{Semi-supervision with MixMatch}

We train semi-supervised scNym models using the MixMatch framework [Berthelot et al., 2019], treating the target dataset as unlabeled data $\mathcal{U}$. At each iteration, MixMatch samples minibatches from both the labeled dataset $(\mathbf{X}, \mathbf{y}) \sim \mathcal{D}$ and unlabeled dataset $\mathbf{U} \sim \mathcal{U}$. We generate "pseudolabels" [Lee, 2013 using model predictions for each observation in the unlabeled minibatch (Supplemental Methods).

$$
u_{i} \sim \mathbf{U} ; z_{i}=f_{\theta}\left(u_{i}\right)
$$

We next "sharpen" the pseudolabels using a "temperature scaling" procedure [Hinton et al., 2015, Guo et al., 2017] with the temperature parameter $T=0.5$ as a form of entropy minimization (Supplemental Methods). This entropy minimization encourages unlabeled examples to belong to one of the described classes.

We then randomly mix each observation and label/pseudolabel pair in both the labeled and unlabeled minibatches with another observation using MixUp [Zhang et al., 2018]. We allow labeled and unlabeled observations to mix together during this procedure (Supplemental Methods).

$$
\lambda \sim \operatorname{Beta}(\alpha, \alpha)
$$

$$
w_{m}=\operatorname{Mix}_{\lambda}\left(w_{i}, w_{j}\right) ; q_{m}=\operatorname{Mix}_{\lambda}\left(q_{i}, q_{j}\right)
$$

where $\left(w_{i}, q_{i}\right)$ is either a labeled observation and ground truth label $\left(x_{i}, y_{i}\right)$ or an unlabeled observation and the pseudolabel $\left(u_{i}, z_{i}\right)$. This procedure yields a minibatch $\mathbf{X}^{\prime}$ of mixed labeled observations and a minibatch $\mathbf{U}^{\prime}$ of mixed unlabeled observations.

We introduce a semi-supervised interpolation consistency penalty during training in addition to the standard supervised loss. For observations and pseudolabels in the mixed unlabeled minibatch $U^{\prime}$, we penalize the mean squared error (MSE) between the mixed pseudolabels and the model prediction for the mixed observation (Supplemental Methods).

$$
L_{\mathrm{SSL}}\left(\mathbf{U}^{\prime}, f_{\theta}\right)=\mathbb{E}_{u_{m}, z_{m} \sim \mathbf{U}^{\prime}}\left\|f_{\theta}\left(u_{m}\right)-z_{m}\right\|_{2}^{2}
$$

This encourages the model to provide smooth interpolations between observations and their ground truth or pseudolabels, generalizing the decision boundary of the model. We weight this unsupervised loss relative to the supervised crossentropy loss using a weighting function $\lambda_{\mathrm{SSL}}(t) \rightarrow[0,1]$. We initialize this coefficient to $\lambda_{\mathrm{SSL}}=0$ and increase the weight to a final value of $\lambda_{\mathrm{SSL}}=1$ over 100 epochs using a sigmoid schedule (Supplemental Methods). 


$$
L\left(\mathbf{X}^{\prime}, \mathbf{U}^{\prime}, f_{\theta}, t\right)=L_{\mathrm{CE}}\left(\mathbf{X}^{\prime}, f_{\theta}\right)+\lambda_{\mathrm{SSL}}(t) L_{\mathrm{SSL}}\left(\mathbf{U}^{\prime}, f_{\theta}\right)
$$

\section{Domain Adaptation with Domain Adversarial Networks}

We use domain adversarial networks (DAN) as an additional approach to incorporate information from the target dataset during training [Ganin et al., 2016]. The DAN method encourages the classification model to embed cells from the training and target dataset with similar coordinates, such that training and target datasets are well-mixed in the embedding. By encouraging the training and target dataset to be well-mixed, we take advantage of the inductive bias that cell identity classes in each dataset are similar, despite technical variation or differences in conditions (Supplemental Methods).

We introduce this technique into scNym by adding an adversarial domain classification network $g_{\phi}$. We implement $g_{\phi}$ as a two-layer neural network with a single hidden layer of 256 units and a rectified linear unit activation, followed by a classification layer with two outputs and a softmax activation. This adversary attempts to predict the domain of origin $d$ from the penultimate classifier embedding $v$ of each observation. For each forward pass, it outputs a probability vector $\hat{d}$ estimating the likelihood the observation came from the training or target domain.

We assign a one-hot encoded domain label $d$ to each molecular profile based on the experiment of origin (Supplemental Methods). During training, we pass a minibatch of labeled observations $x \in \mathbf{X}$ and unlabeled observations $u \in \mathbf{U}$ through the domain adversary to predict domain labels.

$$
\hat{d}=g_{\phi}(v)=g_{\phi}\left(f_{\theta}(x)^{(l-1)}\right)
$$

where $\hat{d}$ is the domain probability vector and $v=f_{\theta}(x)^{(l-1)}$ denotes the embedding of $x$ from the penultimate layer of the classification model $f_{\theta}$. We fit the adversary using a multi-class cross-entropy loss, as described above for the main classification loss (Supplemental Methods).

To make use of the adversary for training the classification model, we use the "gradient reversal" trick at each backward pass. We update the parameters $\phi$ of the adversary using standard gradient descent on the loss $L_{\text {adv }}$. At each backward pass, this optimization improves the adversarial domain classifier (Supplemental Methods). We update the parameters $\theta$ of the classification model using the inverse of the gradients computed during a backward pass from $L_{\text {adv }}$. Using the inverse gradients encourages the classification model $f_{\theta}$ to generate an embedding where it is difficult for the adversary to predict the domain (Supplemental Methods). Our update rule for the classification model parameters therefore becomes:

$$
\theta_{t}=\theta_{t-1}-\eta\left(\frac{\partial L_{\mathrm{CE}}}{\partial \theta}+\lambda_{\mathrm{SSL}}(t) \frac{\partial L_{\mathrm{SSL}}}{\partial \theta}-\lambda_{\mathrm{adv}}(t) \frac{\partial L_{\mathrm{adv}}}{\partial \theta}\right)
$$


We increase the weight of the adversary gradients from $\lambda_{\text {adv }} \rightarrow[0,0.1]$ over the course of 20 epochs during training using a sigmoid schedule. We scale the adversarial gradients flowing to $\theta$, rather than the adversarial loss term, so that full magnitude gradients are used to train a robust adversary $g_{\phi}$ (Supplemental Methods). Incorporating both MixMatch and the domain adversary, our full loss function becomes:

$$
L\left(\mathbf{X}, \mathbf{U}^{\prime}, \mathbf{X}^{\prime}, \mathbf{U}^{\prime}, f_{\theta}, g_{\phi}, t\right)=L_{\mathrm{CE}}\left(\mathbf{X}^{\prime}, f_{\theta}\right)+\lambda_{\mathrm{SSL}}(t) L_{\mathrm{SSL}}\left(\mathbf{U}^{\prime}, f_{\theta}\right)+L_{\mathrm{adv}}\left(\mathbf{X}, \mathbf{U}, f_{\theta}, g_{\phi}, t\right)
$$

\section{Pseudolabel Thresholding for New Cell Type Discovery}

Entropy minimization and domain adversarial training enforce an inductive bias that all cells in the target dataset belong to a class in the training dataset. For many cell type classification tasks, this assumption is valid and useful. However, it is violated in the case where new, unseen cell types are present in the target dataset. We introduce an alternative training configuration to allow for quantitative identification of new cell types in these instances.

We have observed that new cell types will receive low confidence pseudolabels, as they do not closely resemble any of the classes in the training set (Fig. S12). We wish to exclude these low confidence pseudolabels from our entropy minimization and domain adversarial training procedures, as these methods both incorrectly encourage these new cell types to receive high confidence predictions and embeddings for a known cell type. We therefore adopt a notion of "pseudolabel confidence thresholding" introduced in the FixMatch method [Sohn et al., 2020]. To identify confident pseudolabels to use during training, we set a minimum pseudolabel confidence $\tau=0.9$ and assign all pseudolabels a binary confidence indicator $c_{i} \in\{0,1\}$ (Supplemental Methods).

We make two modifications to the training procedure to prevent low confidence pseudolabels from contributing to any component of the loss function. First, we use only high confidence pseudolabels in the MixUp operation of the MixMatch procedure. This prevents low confidence pseudolabels from contributing to the supervised classification or interpolation consistency losses (Supplemental Methods). Second, we use only unlabeled examples with high confidence pseudolabels to train the domain adversary. These low confidence unlabeled examples can therefore occupy a unique region in the model embedding, even if they are easily discriminated from training examples. Our adversarial loss is slightly modified to penalize domain predictions only on confident samples in the pseudolabeled minibatch (Supplemental Methods).

We found that this pseudolabel thresholding configuration option was essential to provide accurate, quantitative information about the presence of new cell types in the target dataset (Fig. S13). However, this option does modestly decrease performance when new cell types are not present. We therefore enable this option when the possibility of new cell types violates the assumption that the training and target data share the same set of cell types. We have provided a simple toggle in our software implementation to allow users to enable or disable this feature. 


\section{scNym Model Embeddings}

We generate gene expression embeddings from our scNym model by extracting the activations of the penultimate neural network layer for each cell. We visualize these embeddings using UMAP [McInnes et al., 2020, Becht et al., 2018] by constructing a nearest neighbor graph $(k=30)$ in principal component space derived from the penultimate activations. We set min_dist $=0.3$ for the UMAP minimum distance parameter.

We present single cell experiments using a 2-dimensional representation fit using the UMAP alogrithm [Becht et al., 2018]. For each experiment, we compute a PCA projection on a set of highly variable genes after $\log (\mathrm{CPM}+1)$ normalization. We construct a nearest neighbor graph using first 50 principal components and fit a UMAP projection from this nearest neighbor graph.

\section{Entropy of Mixing}

We compute the "entropy of mixing" to determine the degree of domain adaptation between training and target datasets in an embedding $X$. The entropy of mixing is defined as the entropy of a vector of class membership in a local neighborhood of the embedding:

$$
H\left(p^{\text {Local }}\right)=-\sum_{k=1}^{K} p_{k}^{\text {Local }} \log p_{k}^{\text {Local }}
$$

where $p^{\text {Local }}$ is a vector of class proportions in a local neighborhood and $k \in K$ are class indices. We compute the entropy of mixing for an embedding $X$ by randomly sampling $n=1000$ cells, and computing the entropy of mixing on a vector of class proportions for the 100 nearest neighbors to each point.

\section{Integrated Gradient Analysis}

We interpreted the predictions of our $\mathrm{scNym}$ models by performing integrated gradient analysis [Sundararajan et al., 2017]. Given a trained model $f_{\theta}$ and a target class $k$, we computed an integrated gradient score IG as the sum of gradients on a class probability $f_{\theta}(x)_{k}$ with respect to an input gene expression vector $x$ at $M=100$ points along a linear path between the zero vector and the input $x$. We then multiplied the sum of gradients for each gene by the expression values in the input $x$. Stated formally, we computed:

$$
\mathrm{IG}\left(x, k, f_{\theta}\right)=x \cdot \frac{1}{M} \sum_{m=1}^{M} \frac{\partial f_{\theta}\left(\frac{m}{M} x\right)_{k}}{\partial x}
$$

In the original integrated gradient formalism, this is equivalent to using the zero vector as a baseline. We average the integrated gradients across $n_{s}$ cell input vectors $x$ to obtain class-level maps $\mathrm{IG}_{k}$, where $n_{s}=\min \left(300, n_{k}\right)$ and $n_{k}$ is the number of cells in the target class. To identify genes that drive incorrect classifications, we computed integrated gradients with respect to some class $k$ for cells with true class $k^{\prime}$ that were incorrectly classified as class $k$. 


\section{Interpretability Comparison}

We compared the biological relevance of features selected by scNym and SVM as a baseline by computing cell type specific Gene Ontology enrichments. We trained both scNym and an SVM to transfer labels from the Tabula Muris 10x Genomics dataset to the Tabula Muris Smart-seq2 dataset. We then extracted feature importance scores from the scNym model using integrated gradients and from the SVM model based on coefficient weights. We selected cell type markers for each model as the top $k=100$ genes with the highest integrated gradient values or SVM coefficients.

For 19 cell types with corresponding Gene Ontology terms, we computed the enrichment of the relevant cell type specific Gene Ontology terms in scNym-derived and SVM-derived cell type markers using Fischer's exact test (Supplemental Methods). We present a sample of the gene sets used (Table S4). We compared the mean Odds-Ratio from Fischer's exact test across relevant Gene Ontology terms between scNym-derived markers and SVM-derived markers. To determine statistical significance of a difference in these mean Odds-Ratios, we performed a paired $t$-test across cell types. We performed the procedure above using $k \in\{50,100,150\}$ to determine the sensitivity of our results to this parameter. We found that scNym integrated gradients had consistently stronger enrichments for relevant Gene Ontology terms across cell types for all values of $k$.

\section{Model Calibration Analysis}

We evaluated scNym calibration by binning all cells in a query set based on the softmax probability of their assigned class $-\max _{k}\left(\operatorname{softmax}\left(f_{\theta}(x)_{k}\right)\right)$ - which we term the "confidence score". We grouped cells into $M=10$ bins $B_{m}$ of equal width from $[0,1]$ and computed the mean accuracy of predictions within each bin.

$$
\begin{aligned}
& \operatorname{acc}\left(B_{m}\right)=\langle\mathbb{1}(\hat{y} \equiv y)\rangle \\
& \operatorname{conf}\left(B_{m}\right)=\left\langle\max \hat{p}_{i}\right\rangle
\end{aligned}
$$

where $\mathbb{1}(a \equiv b)$ denotes a binary equivalency operation that yields 1 if $a$ and $b$ are equivalent and 0 otherwise and $\langle\cdot\rangle$ denotes the arithmetic average.

We computed the "expected calibration error" as previously proposed [Thulasidasan et al., 2019].

$$
\mathrm{ECE}=\sum_{m=1}^{M} \frac{\left|B_{m}\right|}{N}\left|\operatorname{acc}\left(B_{m}\right)-\operatorname{conf}\left(B_{m}\right)\right|
$$

We also computed the "overconfidence error", which specifically focuses on high confidence but incorrect predictions.

$$
\operatorname{oe}\left(B_{m}\right)=\operatorname{conf}\left(B_{m}\right) \max \left(\left(\operatorname{conf}\left(B_{m}\right)-\operatorname{acc}\left(B_{m}\right)\right), 0\right)
$$




$$
\mathrm{OE}=\sum_{m=1}^{M} \frac{\left|B_{m}\right|}{N} \mathrm{oe}\left(B_{m}\right)
$$

where $N$ is the total number of samples, and $\left|B_{m}\right|$ is the number of samples in bin $B_{m}$.

We performed this analysis for each model trained in a 5-fold cross-validation split to estimate calibration for a given model configuration. We evaluated calibrations for baseline neural network models, models with MixUp but not MixMatch, and models with the full MixMatch procedure.

\section{Baseline Methods}

As baseline methods, we used ten cell identity classifiers: scmap-cell, scmap-cluster [Kiselev et al., 2018, Andrews and Hemberg, 2018], scmap-cell-exact (scmap-cell with exact k-NN search), a linear SVM [Abdelaal et al., 2019], scPred [Alquicira-Hernandez et al., 2019], singleCellNet [Tan and Cahan, 2019], CHETAH [de Kanter et al., 2019], Harmony followed by an SVM [Korsunsky et al., 2019], LIGER followed by an SVM [Stuart et al., 2019], and scANVI [Lopez et al., 2018, Xu et al., 2019]. For model training, we split data into 5-folds and trained five separate models, each using 4 folds for training and validation data. This allowed us to assess variation in model performance as a function of changes in the training data. No class balancing was performed prior to training, though some methods perform class balancing internally. All models, including scNym, were trained on the same 5-fold splits to ensure equitable access to information. All methods were run with the best hyperparameters suggested by the authors unless otherwise stated for our hyperparameter optimization comparisons (full details in Supplemental Methods).

We applied all baseline methods to all benchmarking tasks. If a method could not complete the task given $256 \mathrm{~GB}$ of RAM and 8 CPU cores, we reported the accuracy for that method as "Undetermined." Only scNym and scANVI models required GPU resources. We trained models on Nvidia K80, GTX1080ti, Titan RTX, or RTX 8000 GPUs, using only a single GPU per model.

\section{Performance Benchmarking}

For all benchmarks, we computed the mean accuracy across cells (“Accuracy”), Cohen's $\kappa$-score, and the multiclass recevier operating characteristic (MCROC). We computed the MCROC as the mean of ROC scores across cell types, treating each cell type as a binary classification problem. We performed quality control filtering and pre-processing on each dataset before training (Supplemental Methods).

For the Rat Aging Cell Atlas [Ma et al., 2020] benchmark, we trained scNym models on single cell RNA-seq from young, ad libitum fed rats (5 months old) and predicted on cells from aged rats (ad libitum fed or calorically-restricted). For the human PBMC stimulation benchmark, we trained models on unstimulated PBMCs collected from multiple human donors and predicted on IFNB1 stimulated PBMCs collected in the same experiment [Kang et al., 2017]. 
For the Tabula Muris cross-technology benchmark, we trained models on Tabula Muris 10x Genomics Chromium platform and predicted on data generated using Smart-seq2. For the Mouse Cell Atlas (MCA) [Han et al., 2018] benchmark, we trained models on single cell RNA-seq from lung tissue in the Tabula Muris 10x Chromium data [Tabula Muris Consortium, 2018] and predicted on MCA lung data. For the spatial transcriptomics benchmark, we trained models on spatial transcriptomics from a mouse sagittal-posterior brain section and predicted labels for another brain section (data downloaded from https://www.10xgenomics.com/resources/datasets/

For the single cell to single nucleus benchmark in the mouse kidney, we trained scNym models on all single cell data from six unique sequencing protocols and predicted labels for single nuclei from three unique protocols [Denisenko et al., 2020]. For the single nucleus to single cell benchmark, we inverted the training and target datasets above to train on the nuclei datasets and predict on the single cell datasets. We set unique domain labels for each protocol during training in both benchmark experiments. To evaluate the impact of multi-domain training, we also trained models on only one single cell or single nucleus protocol using the domains from the opposite technology as target data.

For the multi-domain cross-technology benchmark in mouse cortex nuclei, we generated four distinct subtasks from data generated using four distinct technologies to profile the same samples [Ding et al., 2020]. We trained scNym and baseline methods to predict labels on one technology given the remaining three technologies as training data for all possible combinations. We used each technology as a unique domain label for scNym.

For the cross-species mouse to rat demonstration, we selected a set of cell types with comparable annotations in the Tabula Muris and Rat Aging Cell Atlas [Ma et al., 2020] to allow for quantitative evaluation. We trained scNym with mouse data as the source domain and rat data as the target domain. We used the new identity discovery configuration to account for the potential for new cell types in a cross-species experiment. For the cross-species mouse to human demonstration, we similarly selected a set of cell types with comparable cell annotation ontologies in the Tabula Muris 10x lung data and human lung cells from the IPF Cell Atlas [Habermann et al., 2020]. We trained an scNym model using mouse data as the source domain and human data as the target, as for the mouse to rat demonstration.

\section{Runtime Benchmarking}

We measured the runtime of scNym and each baseline classification method using subsamples from the multi-domain kidney single cell and single nuclei dataset [Denisenko et al., 2020]. We measured runtimes for annotation transfer from single cells to single nuclei labels using subsamples of size $n \in\{1250,2500,5000,10000,20000,40000\}$ for each of the training and target datasets. All methods were run on four cores of a $2.1 \mathrm{GHz}$ Intel Xeon Gold $6130 \mathrm{CPU}$ and 64 GB of CPU memory. GPU capable methods (scNym, scANVI) were provided with one Nvidia Titan RTX GPU (consumer grade CUDA compute device). 


\section{Hyperparameter Optimization Experiments}

We performed hyperparameter optimization across four tasks for the top three baseline methods, the SVM, singleCellNet, and scmap-cell-exact. For the SVM, we optimized the regularization strength parameter $C$ at 12 values $\left(C \in 10^{k} \forall k \in\right.$ $[-6,5])$ with and without class weighting. For class weighting, we set class weights as either uniform or inversely proportional to the number of cells in each class to enforce class balancing $\left(w_{k}=1 / n_{k}\right.$, where $w_{k}$ is the weight for class $k$ and $n_{k}$ is the number of cells for that class). For scmap-cell-exact, we optimized (1) the number of nearest neighbors $(k \in\{5,10,30,50,100\}),(2)$ the distance metric $(d(\cdot, \cdot) \in\{$ cosine, euclidean $\}$ ), and (3) the number of features to select with M3Drop $\left(n_{f} \in\{500,1000,2000,5000\}\right.$ ). For singleCellNet, we optimized with nTopGenes $\in\{10,20\}$, nRand $\in\{35,70,140\}$, nTrees $\in\{100,1000,2000\}$, and nTopGenePairs $\in\{12,25\}$.

We optimized scNym for two of the four tasks, due to computational expense and superiority of default parameters relative to baseline methods. For scNym, we optimized (1) weight decay $\left(\lambda_{w} \in 10^{-5}, 10^{-4}, 10^{-3}\right)$, (2) batch size $(M \in\{128,256\})$, (3) the number of hidden units ( $h \in\{256,512\})$, (4) the maximum MixMatch weight $\left(\lambda_{\mathrm{SSL}} \in\{0.01,0.1,1.0\}\right)$, and (5) the maximum DAN weight $\left(\lambda_{\mathrm{Adv}} \in\{0.01,0.1,0.2\}\right)$. We did not optimize weight decay for the PBMC cross-stimulation task. We performed a grid search for all methods.

Hyperparameter optimization is non-trivial in the context of a domain shift between the training and test set. Traditional optimization using cross-validation on the training set alone may overfit parameters to the training domain, leading to suboptimal outcomes. This failure mode is especially problematic for domain adaptation models, where decreasing the strength of domain adaptation regularizers may improve performance within the training data, while actually decreasing performance on the target data.

In light of these concerns, we adopted a procedure known as reverse cross-validation to evaluate each hyperparameter set [Zhong et al., 2010]. Reverse cross-validation uses both the training and target datasets during training to account for the effect of hyperparameters on the effectiveness of transferring labels across domains. Formally, we first split the labeled training data $\mathcal{D}$ into a training set, validation set, and held-out test set $\mathcal{D}^{\prime}, \mathcal{D}^{v}, \mathcal{D}^{*}$. We use $10 \%$ of the training dataset for the validation set and $10 \%$ for the held-out test set. We then train a model $f_{\theta}: x \rightarrow \hat{y}$ to transfer labels from the training set $\mathcal{D}^{\prime}$ to the target data $\mathcal{U}$. We use the validation set $\mathcal{D}^{v}$ for early stopping with scNym and concatenate it into the training set for other methods that do not use a validation set. We treat the predictions $\hat{y}=f_{\theta}(u)$ as pseudolabels for the unlabeled dataset and subsequently train a second model $f_{\phi}: u \rightarrow \tilde{y}$ to transfer annotations from the "pseudolabeled" dataset $\mathcal{U}$ back to the labeled dataset $\mathcal{D}$. We then evaluate the "reverse accuracy" as the accuracy of the labels $\tilde{y}$ for the held-out test portion of the labeled dataset, $\mathcal{D}^{*}$.

We performed this procedure using a standard 5-fold split for each parameter set. We computed the mean reverse cross-validation accuracy as the performance metric for robustness. For each method that we optimized, we selected the optimal set of hyperparameters as the set with the top reverse cross-validation accuracy. 


\section{New Cell Type Discovery Experiments}

\section{New Cell Type Discovery with Pre-trained Models}

We evaluated the ability of scNym to highlight new cell types, unseen in the training data by predicting cell type annotations in the Tabula Muris brain data (Smart-seq2) using models trained on the 10x Genomics data from the ten tissues noted above with the Smart-seq2 data as corresponding target dataset. No neurons or glia were present in the training or target set for this experiment. This experiment simulates the scenario where a pre-trained model has been fit to transfer across technologies (10x to Smart-seq2) and is later used to predict cell types in a new tissue, unseen in the original training or target data.

We computed scNym confidence scores for each cell as $c_{i}=\max p_{i}$, where $p_{i}$ is the model prediction probability vector for cell $i$ as noted above. To highlight potential cell type discoveries, we set a simple threshold on these confidence scores $d_{i}=c_{i} \leq 0.5$, where $d_{i} \in\{0,1\}$ is a binary indicator variable. We found that scNym assigned low confidence to the majority of cells from newly "discovered" types unseen in the training set using this method.

\section{New Cell Type Discovery with Semi-supervised Training}

We also evaluated the ability of scNym to discover new cell types in a scenario where new cell types are present in the target data used for semi-supervised training. We used the same training data and target data as the experiment above, but we now introduce the Tabula Muris brain data (Smart-seq2) into the target dataset during semi-supervised training. We performed this experiment using our default scNym training procedure, as well as the modified new cell type discovery procedure described above.

As above, we computed confidence scores for each cell and set a threshold of $d_{i}=c_{i} \leq 0.5$ to identify potential new cell type discoveries. We found that scNym models trained with the new cell type discovery procedure provided low confidence scores to the new cell types, suitable for identification of these new cells. We considered all new cell type predictions to be incorrect when computing accuracy for the new cell type discovery task.

\section{Clustering Candidate New Cell Types}

We employed a community detection procedure in the scNym embedding to suggest the number of distinct cell states represented by low confidence cells. First, we identify cells with a confidence score lower than a threshold $t_{\text {conf }}$ to highlight putative cell type discoveries, $d_{i}=c_{i}<t_{\text {conf }}$. We then extract the scNym penultimate embedding activations for these low confidence cells and construct a nearest neighbor graph using the $k=15$ nearest neighbors for each cell. We compute a Leiden community detection partition for a range of different resolution parameters $r \in\{0.1,0.2,0.3,0.5,1.0\}$ and compute the Calinski-Harabasz score for each partition [Calinski and Harabasz, 1974]. We select the optimal partition in the scNym embedding as the partition generated with the maximum Calinski-Harabasz score and suggest that communities in this partition may each represent a distinct cell state. 


\section{Discriminating Candidate New Cell Types from Other Low Confidence Predictions}

Cells may receive low confidence predictions for multiple reasons, including: (1) a cell is on the boundary between two cell types, (2) a cell has very little training data for the predicted class, and (3) the cell represents a new cell type unseen in the training dataset. To discriminate between these possibilities, we employ a heuristic similar to the one we use for proposing a number of new cell types that might be present. First, we extract the scNym embedding coordinates from the penultimate layer activations for all cells and build a nearest neighbor graph. We then optimize a Leiden cluster partition by scanning different resolution parameters to maximize the Calinksi-Harabasz score. We then compute the average prediction confidence across all cells in each of the resulting clusters. We also visualize the number of cells present in the training data for each predicted cell type.

We consider cells with low prediction scores within an otherwise high confidence cluster to be on the boundary between cell types. These cells may benefit from domain expert review of the specific criteria to use when discriminating between very similar cell identities. We consider low confidence cell clusters with few training examples for the predicted class to warrant further domain expert review. Low confidence clusters that are predicted to be a class with ample training data may represent new cell types and also warrant further review.

\section{Software Availability}

Open source code for our software and pre-processed reference datasets analyzed in this study are available in the scNym repository (https://github.com/calico/scnym) and as Supplemental Code.

\section{Competing Interests}

JCK and DRK are paid employees of Calico Life Sciences, LLC.

\section{Acknowledgements}

JCK conceived the study, implemented software, conducted experiments, and wrote the paper. DRK conceived the study and wrote the paper. We thank Zhenghao Chen, Amoolya H. Singh, and Han Yuan for helpful discussions and comments. Funding for this study was provided by Calico Life Sciences, LLC. 
${ }_{521}$ Figures

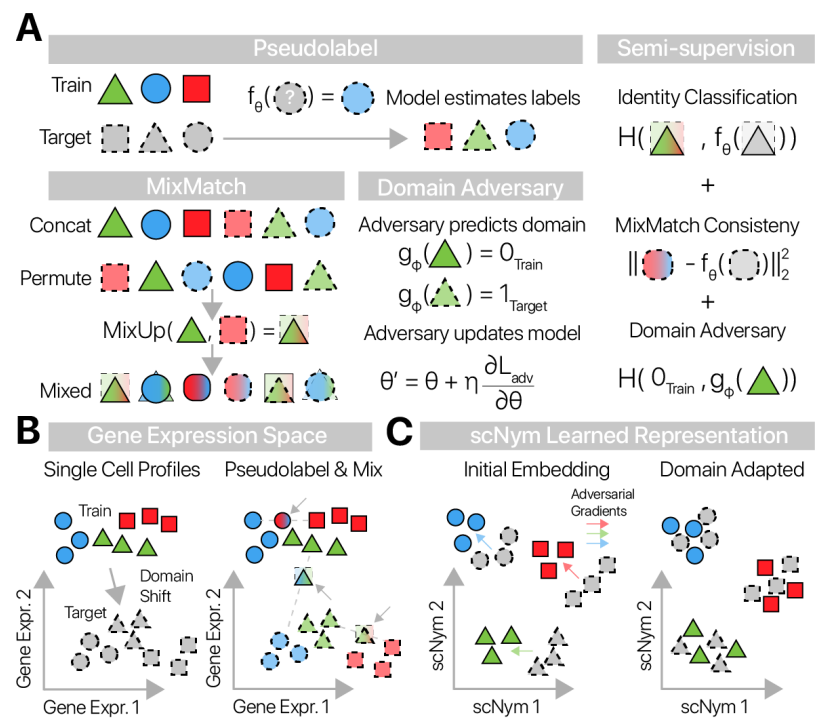

Figure 1 

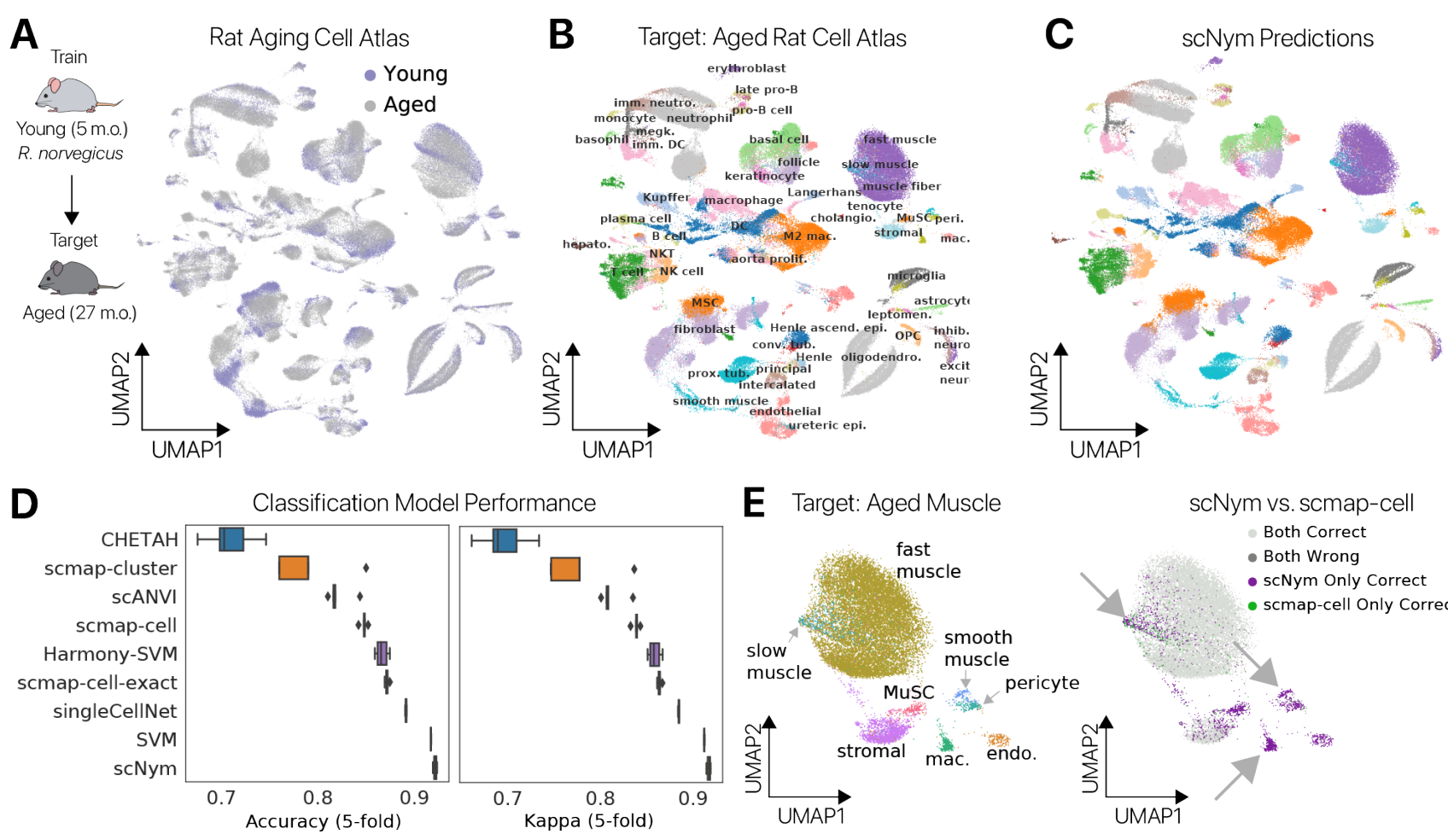

E Target: Aged Muscle

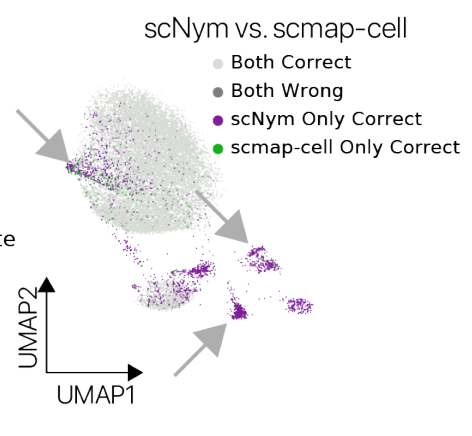

Figure 2
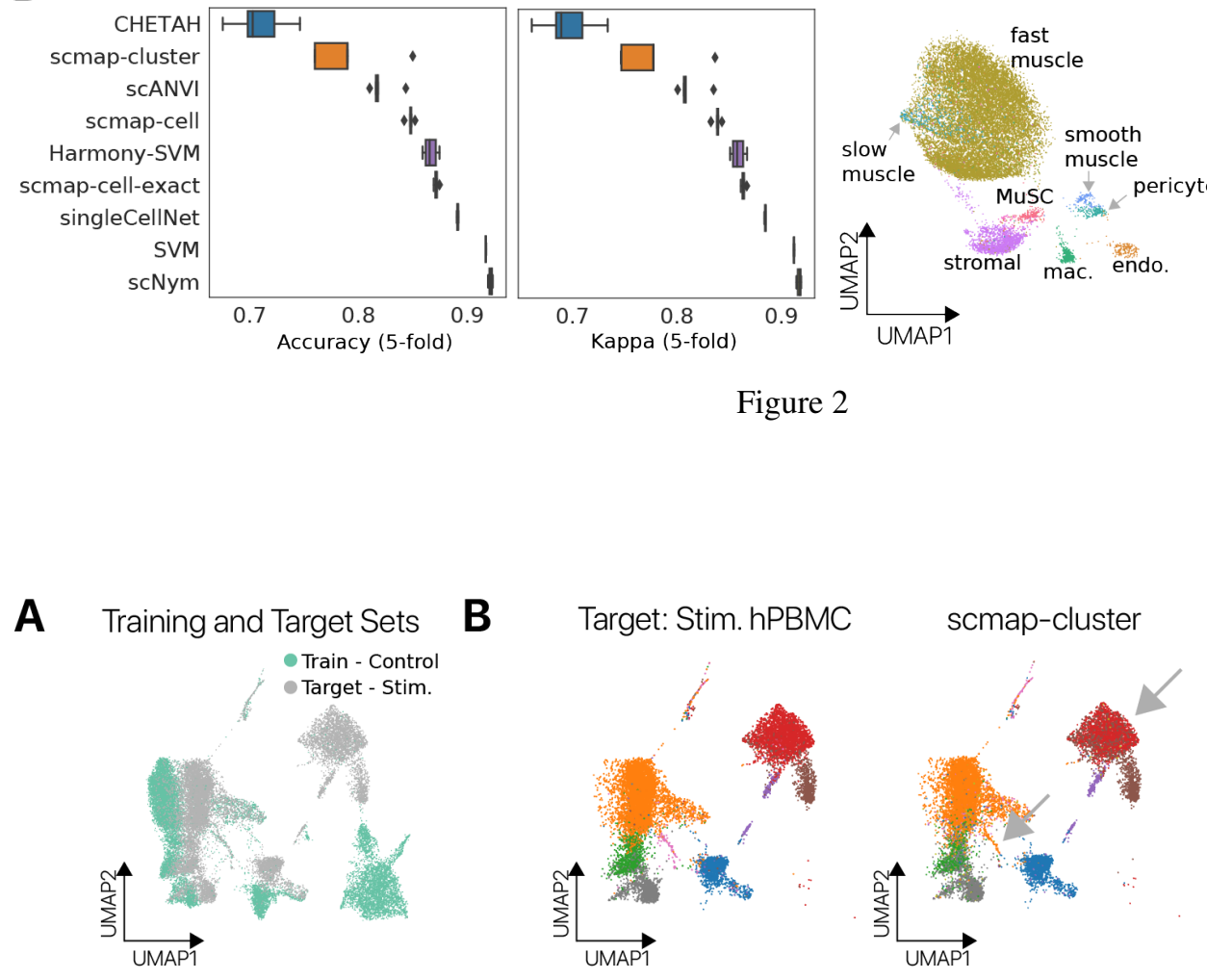

.
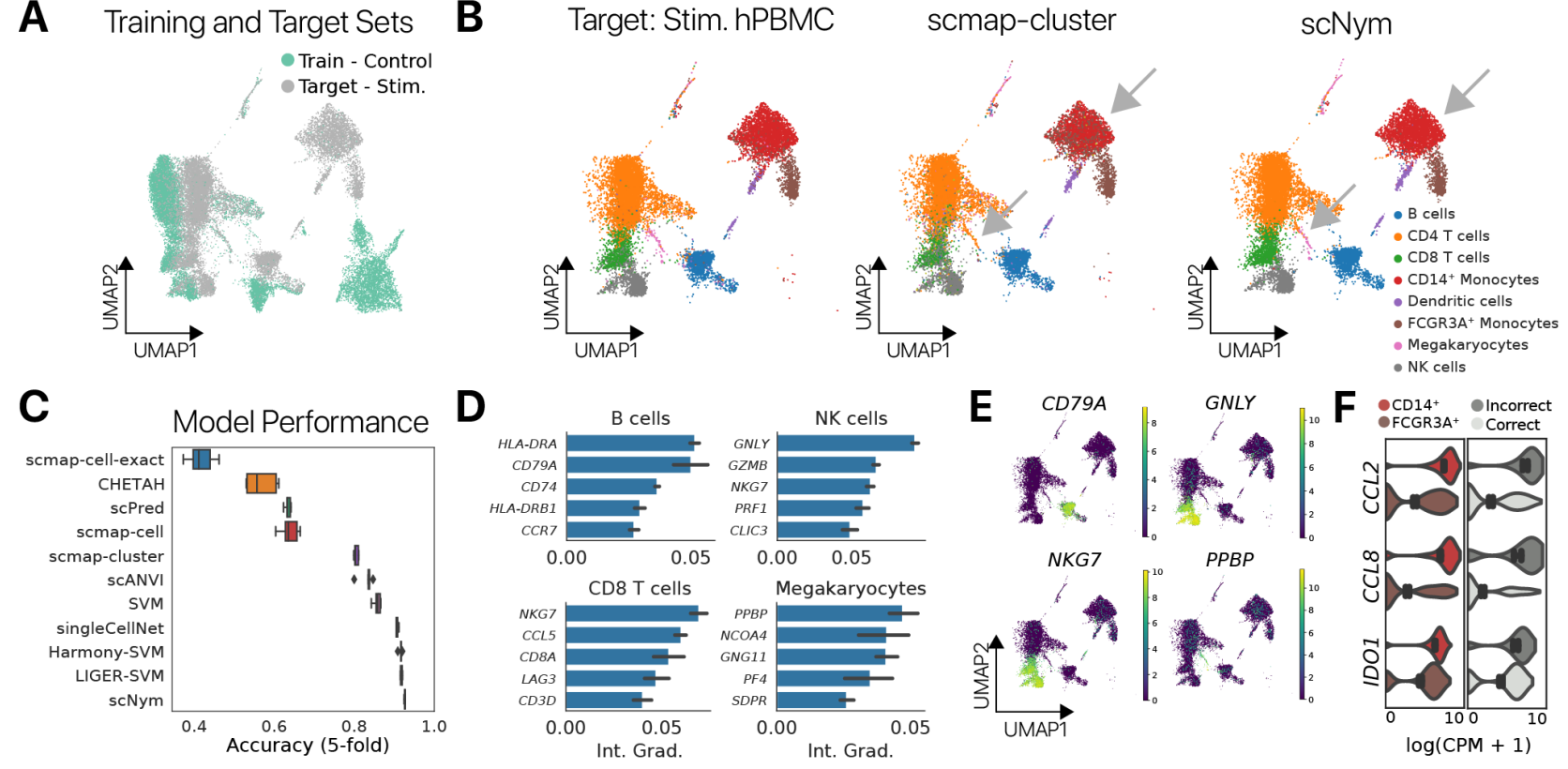

Figure 3 


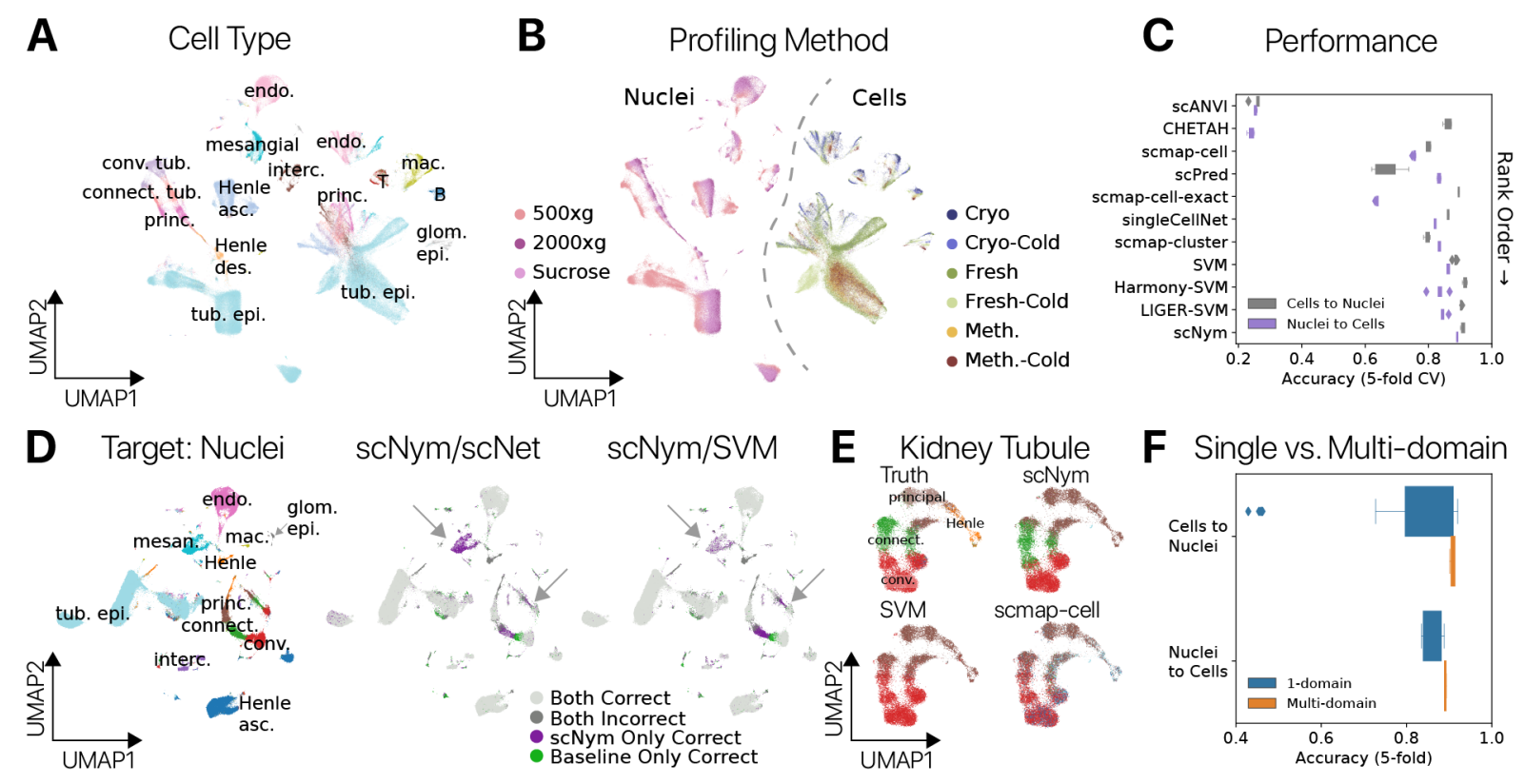

Figure 4

A

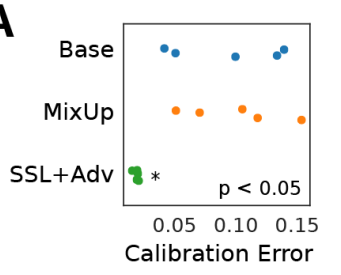

C Target: Brain Cells

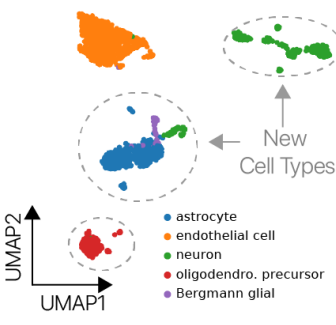

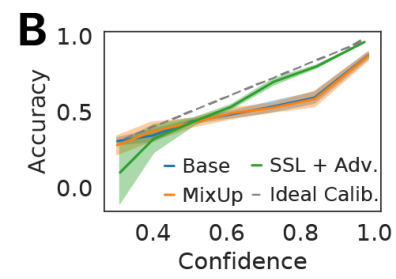

D scNym Confidence

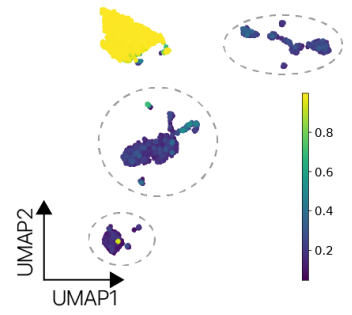

Figure 5 


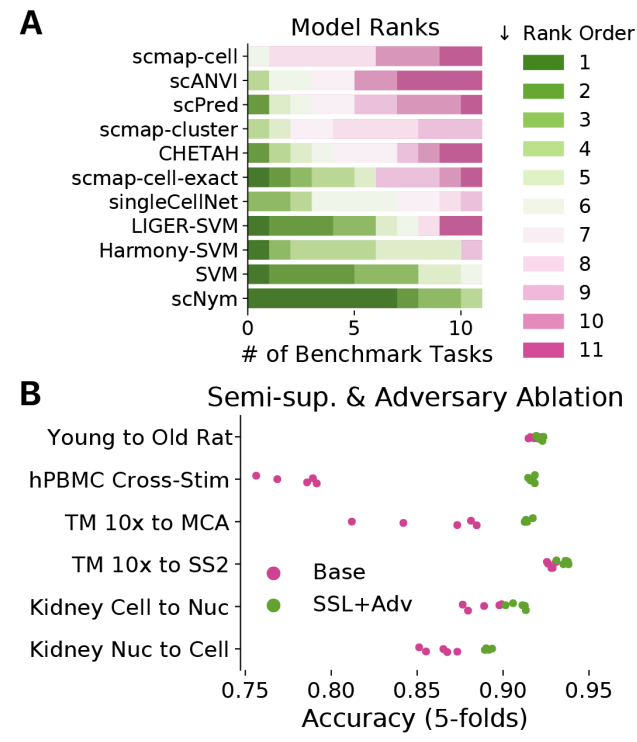

Figure 6 


\section{Figure Legends}

Figure 1: scNym combines semi-supervised and adversarial training to learn performant single cell classifiers. (A) scNym takes advantage of target data during training by estimating "pseudolabels" for each target data point using model predictions. Training and target cell profiles and their labels are then augmented using weighted averages in the MixMatch procedure. An adversary is also trained to discriminate training and target observations. We train model parameters using a combination of supervised classification, interpolation consistency, and adversarial objectives. Here, we use $H(\cdot, \cdot)$ to represent the cross-entropy function. (B) Training and target cell profiles are separated by a domain shift in gene expression space. scNym pseudolabels target profiles and generates mixed cell profiles (arrows) by randomly pairing cells. Mixed profiles form a bridge between training and target datasets. (C) scNym models learn a discriminative representation of cell state in a hidden embedding layer. Train and target cell profiles initially segregate in this representation. During training, adversarial gradients (colored arrows) encourage cells of the same type to mix in the scNym embedding.

Figure 2: scNym transfers cell identity annotations between young and aged rat cells. (A) Young and aged cells from a rat aging cell atlas displayed in a UMAP projection [Ma et al., 2020]. Some cell types show a domain shift between young and aged cells. scNym models were trained on young cells in the atlas and used to predict labels for aged cells. (B) Ground truth cell type annotations for the aged cells of the Rat Aging Cell Atlas shown in a UMAP projection. (C) scNym predicted cell types in the target aged cells. scNym predictions match ground truth annotation in the majority $(>90 \%)$ of cases. (D) Accuracy (left) and $\kappa$-scores (right) for scNym and other state of the art classification models. scNym yields significantly greater accuracy and $\kappa$-scores than baseline methods $(p<0.01$, Wilcoxon Rank Sums). Note: multiple existing methods could not complete this large task. (E) Aged skeletal muscle cells labeled with ground truth annotations (left) and the relative accuracy of scNym and scmap-cell (right) projected with UMAP. scNym accurately predicts multiple cell types that are confused by scmap-cell (arrows).

Figure 3: scNym transfers annotations from unstimulated immune cells to stimulated immune cells. (A) UMAP projection of unstimulated PBMC training data and stimulated PBMC target data with stimulation condition labels. (B) UMAP projections of ground truth cell type labels (left), scmap-cluster predictions (center), and scNym predictions (right). scNym provides consistent annotations for both $\mathrm{CD}_{14}{ }^{+}$and $\mathrm{FCGR} \mathrm{A}^{+}$monocytes. scmap-cluster confuses these populations (arrow). (C) Classification accuracy for scNym and baseline cell identity classification methods. scNym is significantly more accurate than other approaches $(p<0.01$, Wilcoxon Rank Sums). (D) Integrated gradient analysis reveals genes that drive correct classification decisions. We recover known marker genes of many cell types (e.g. $C D 79 A$ for B cells, PPBP for megakaryocytes). (E) Cell type specificity of the top salient genes in a UMAP projection of gene expression (log normalized counts per million). (F) Integrated gradient analysis reveals genes that drive incorrect classification of some FCGR3A ${ }^{+}$monocytes as CD14 ${ }^{+}$monocytes. Several of the top 15 salient genes for misclassification are $\mathrm{CD}_{1}{ }^{+}$markers that are upregulated in incorrectly classified $\mathrm{FCGR}^{+} \mathrm{A}^{+}$cells. 
Figure 4: Multi-domain training improves cross-technology annotation transfer in the mouse kidney. (A) Cell type and (B) sequencing protocol annotations in a UMAP projection of single cell and nucleus RNA-seq profiles from the mouse kidney [Denisenko et al., 2020]. Each protocol represents a unique training domain that captures technical variation. (C) Performance of scNym and baseline approaches on single cell to nucleus and single nucleus to cell annotation transfer. Methods are rank ordered by performance across tasks. scNym is superior to each baseline method on at least one task (Wilcoxon Rank Sum, $p<0.05$ ). (D) Single nucleus target data labeled with true cell types (left) or the relative accuracy of scNym and baseline methods (right) for the single cell to single nucleus task. scNym achieves more accurate labeling of mesangial cells and tubule cell types (arrows). (E) Kidney tubule cells from (D) visualized independently with true and predicted labels. scNym offers the closest match to true annotations. All methods make notable errors on this difficult task. (F) Comparison of scNym performance when trained on individual training datasets (1-domain) vs. multi-domain training across all available datasets. We found that multi-domain training improves performance on both the cells to nuclei and nuclei to cells transfer tasks (Wilcoxon Rank Sums, $p=0.073$ and $p<0.01$ respectively).

Figure 5: scNym confidence scores highlight unseen cell types. (A) scNym calibration error for models trained on the human PBMC cross-stimulation task. Semi-supervised and adversarial training significantly reduced calibration error relative to models trained with only supervised methods (Base, MixUp). (B) Calibration curves capturing the relationship between model confidence and empirical accuracy for models in (A). (C) scNym models were trained to transfer annotations from a mouse atlas without brain cell types to data from mouse brain tissue. We desire a model that provides low confidence scores to the new cell types and high confidence scores for endothelial cells seen in other tissues. (D) scNym confidence scores for target brain cells. New cell types receive low confidence scores as desired (dashed outlines).

Figure 6: Comparison of semi-supervised scNym to other single cell classification methods and ablated scNym variants. (A) We assign each method a rank order (Rank 1 is best) based on performance for each benchmark task. scNym is the top ranked method across tasks and ranks highly on all tasks. A support vector machine (SVM) baseline is the next best method, consistent with a previous benchmarking study [Abdelaal et al., 2019]. (B) Ablation experiments comparing simplified supervised scNym models (Base) against the full scNym model with semi-supervised and adversarial training (SSL + Adv.). We found that semi-supervised and adversarial training significantly improved scNym performance across diverse tasks (all tasks shown, Wilcoxon Rank Sum, $p<0.05$ ). 


\begin{tabular}{|c|c|c|c|c|c|c|c|c|c|c|c|}
\hline & scmap-cell & scmap-cell-exact & scmap-cluster & SVM & singleCellNet & scPred & CHETAH & Harmony-SVM & LIGER-SVM & scANVI & $\mathrm{scNym}$ \\
\hline Young to Old Rat & $84.8 \pm 0.002$ & $87.2 \pm 0.001$ & $79.0 \pm 0.016$ & $91.7 \pm 0.0$ & $9.1 \pm 0.0$ & OOM & $70.9 \pm 0.012$ & $86.6 \pm 0.003$ & OOM & $82.1 \pm 0.006$ & $92.2 \pm 0.001$ \\
\hline hPBM & & & & $.8 \pm 0.004$ & $.8 \pm 0.001$ & $3.4 \pm 0.003$ & & & $91.8 \pm 0.001$ & & \\
\hline TM 10x to MCA & $3.6 \pm 0.005$ & $9.7 \pm 0.001$ & $.3 \pm 0.001$ & $88.4 \pm 0.001$ & $80.5 \pm 0.005$ & $61.2 \pm 0.025$ & $84.7 \pm 0.006$ & $.3 \pm 0.007$ & $38.4 \pm 0$ & $85.9 \pm 0.002$ & $91.4 \pm 0.001$ \\
\hline TM 10 & $62.4 \pm 0.005$ & & & & & & & & & & \\
\hline Spatial & 0.005 & 038 & 0.001 & 0.001 & 0.002 & 0.001 & 0.005 & 05 & 001 & 0.007 & 0.002 \\
\hline Il to Nuc & & & & & & & & & & & 0.002 \\
\hline Kidney Nuc to Cell & $75.6 \pm 0.002$ & $63.8 \pm 0.002$ & $3.5 \pm 0.001$ & $86.3 \pm 0.001$ & $82.1 \pm 0.001$ & $83.3 \pm 0.002$ & $23.9 \pm 0.004$ & $83.4 \pm 0.0$ & $84.8 \pm 0.004$ & \pm 0.008 & $89.1 \pm 0.001$ \\
\hline & & & & & & & & & $85.6 \pm 0.001$ & \pm 0.009 & $86.0 \pm 0.002$ \\
\hline Cortex 10x & $3.5 \pm 0.005$ & $91.1 \pm 0.002$ & $87.4 \pm 0.003$ & $91.3 \pm 0.0$ & 0.002 & $90.5 \pm$ & $93.1=$ & $91.2 \pm$ & $91.1 \pm$ & $=0.021$ & $94.5 \pm 0.002$ \\
\hline Cortex DroNc & $69.2 \pm 0.003$ & $71.3 \pm 0.007$ & $77.0 \pm 0.003$ & $81.5 \pm 0.004$ & $83.3 \pm 0.002$ & $80.3 \pm 0$ & $83.3 \pm$ & $82.2 \pm 0$ & $87.7 \pm$ & $56.4 \pm 0$ & $89.4 \pm 0.002$ \\
\hline Cortex sci-seq & $82.8 \pm 0.002$ & $78.0 \pm 0.001$ & $79.3 \pm 0.001$ & $85.2 \pm 0.001$ & $83.6 \pm 0.001$ & $83.8 \pm 0.002$ & $83.0 \pm 0.001$ & $83.9 \pm 0.003$ & $84.8 \pm 0.007$ & $60.9 \pm 0.014$ & $84.1 \pm 0.002$ \\
\hline
\end{tabular}

Table 1: Comparison of model performance across tasks. Mean accuracy \pm standard error across a 5-fold training split is reported. Bold text marks best models per task $(p<0.05$, Rank Sums test). Multiple bolded models indicates statistically insignificant differences between the bolded models. OOM indicates that the method encountered an out-of-memory error on our hardware (256GB RAM). scNym is the top ranked model across tasks.

\section{References}

Abdelaal T, Michielsen L, Cats D, Hoogduin D, Mei H, Reinders MJT, Mahfouz A, 2019. A comparison of automatic cell identification methods for single-cell RNA sequencing data. Genome Biology 20: 194. doi:10.1186/ s13059-019-1795-z.

Alquicira-Hernandez J, Sathe A, Ji HP, Nguyen Q, Powell JE, 2019. scPred: accurate supervised method for cell-type classification from single-cell RNA-seq data. Genome Biology 20: 264. doi:10.1186/s13059-019-1862-5.

Andrews TS, Hemberg M, 2018. M3Drop: dropout-based feature selection for scRNASeq. Bioinformatics 35: 2865-2867. doi:10.1093/bioinformatics/bty1044.

Angelidis I, Simon LM, Fernandez IE, Strunz M, Mayr CH, Greiffo FR, Tsitsiridis G, Ansari M, Graf E, Strom TM, et al., 2019. An atlas of the aging lung mapped by single cell transcriptomics and deep tissue proteomics. Nat Commun pp. 1-17. doi:10.1038/s41467-019-08831-9.

Becht E, McInnes L, Healy J, Dutertre CA, Kwok IWH, Ng LG, Ginhoux F, Newell EW, 2018. Dimensionality reduction for visualizing single-cell data using UMAP. Nature Biotechnology 37: 38-44. doi:10.1038/nbt.4314.

Berthelot D, Carlini N, Goodfellow I, Papernot N, Oliver A, Raffel CA, 2019. MixMatch: A Holistic Approach to Semi-Supervised Learning. In Advances in Neural Information Processing Systems 32, pp. 5049-5059.

Calinski T, Harabasz J, 1974. A dendrite method for cluster analysis. Communications in Statistics - Theory and Methods 3: 1-27.

Denisenko E, Guo BB, Jones M, Hou R, de Kock L, Lassmann T, Poppe D, Clément O, Simmons RK, Lister R, et al., 2020. Systematic assessment of tissue dissociation and storage biases in single-cell and single-nucleus RNA-seq workflows. Genome Biology 21: 130. doi:10.1186/s13059-020-02048-6.

de Kanter JK, Lijnzaad P, Candelli T, Margaritis T, Holstege FCP, 2019. CHETAH: a selective, hierarchical cell type identification method for single-cell RNA sequencing. Nucleic Acids Research 47: e95. doi:10.1093/nar/gkz543. 
Diehl AD, Meehan TF, Bradford YM, Brush MH, Dahdul WM, Dougall DS, He Y, Osumi-Sutherland D, Ruttenberg A, Sarntivijai S, et al., 2016. The Cell Ontology 2016: enhanced content, modularization, and ontology interoperability. Journal of biomedical semantics 7: 44-10.

Ding J, Adiconis X, Simmons SK, Kowalczyk MS, Hession CC, Marjanovic ND, Hughes TK, Wadsworth MH, Burks T, Nguyen LT, et al., 2020. Systematic comparison of single-cell and single-nucleus RNA-sequencing methods. Nature Biotechnology 38: 737-746. doi:10.1038/s41587-020-0465-8.

Dixit A, Parnas O, Li B, Chen J, Fulco CP, Jerby-Arnon L, Marjanovic ND, Dionne D, Burks T, Raychowdhury R, et al., 2016. Perturb-Seq: Dissecting Molecular Circuits with Scalable Single-Cell RNA Profiling of Pooled Genetic Screens. Cell 167: 1853-1857.e17.

Ganin Y, Ustinova E, Ajakan H, Germain P, Larochelle H, Laviolette F, Marchand M, Lempitsky V, 2016. DomainAdversarial Training of Neural Networks. arXiv:1505.07818 [cs, stat] ArXiv: 1505.07818.

Guo C, Pleiss G, Sun Y, Weinberger KQ, 2017. On Calibration of Modern Neural Networks. In International Conference on Machine Learning, pp. 1-10.

Habermann AC, Gutierrez AJ, Bui LT, Yahn SL, Winters NI, Calvi CL, Peter L, Chung MI, Taylor CJ, Jetter C, et al., 2020. Single-cell RNA sequencing reveals profibrotic roles of distinct epithelial and mesenchymal lineages in pulmonary fibrosis. Science Advances 6: eaba1972. doi:10.1126/sciadv.aba1972.

Han X, Wang R, Zhou Y, Fei L, Sun H, Lai S, Saadatpour A, Zhou Z, Chen H, Ye F, et al., 2018. Mapping the Mouse Cell Atlas by Microwell-Seq. Cell 173: 1307.

Hinton G, Vinyals O, Dean J, 2015. Distilling the Knowledge in a Neural Network. In NIPS Deep Learning and Representation Learning Workshop.

Ioffe S, Szegedy C, 2015. Batch Normalization: Accelerating Deep Network Training by Reducing Internal Covariate Shift. In Proceedings of the 32nd International Conference on Machine Learning, volume 37 of Proceedings of Machine Learning Research, pp. 448-456. PMLR, Lille, France.

Kang HM, Subramaniam M, Targ S, Nguyen M, Maliskova L, McCarthy E, Wan E, Wong S, Byrnes L, Lanata CM, et al., 2017. Multiplexed droplet single-cell RNA-sequencing using natural genetic variation. Nature Biotechnology 36: 89-94.

Kimmel JC, Penland L, Rubinstein ND, Hendrickson DG, Kelley DR, Rosenthal AZ, 2019. Murine single-cell RNA-seq reveals cell-identity- and tissue-specific trajectories of aging. Genome Research 29: 2088-2103.

Kingma DP, Mohamed S, Jimenez Rezende D, Welling M, 2014. Semi-supervised Learning with Deep Generative Models 27: 3581-3589.

Kiselev VY, Yiu A, Hemberg M, 2018. scmap: projection of single-cell RNA-seq data across data sets. Nature methods 15: 359-362. doi:10.1038/nmeth.4644. 
Korsunsky I, Millard N, Fan J, Slowikowski K, Zhang F, Wei K, Baglaenko Y, Brenner M, Loh Pr, Raychaudhuri S, 2019. Fast, sensitive and accurate integration of single-cell data with Harmony. Nature Methods 16: 1289-1296. doi:10.1038/s41592-019-0619-0.

Lee DH, 2013. Pseudo-Label : The Simple and Efficient Semi-Supervised Learning Method for Deep Neural Networks. ICML 2013 Workshop : Challenges in Representation Learning (WREPL) .

Lopez R, Regier J, Cole MB, Jordan MI, Yosef N, 2018. Deep generative modeling for single-cell transcriptomics. Nature methods pp. 1-11. doi:10.1038/s41592-018-0229-2.

Ma S, Sun S, Geng L, Song M, Wang W, Ye Y, Ji Q, Zou Z, Wang S, He X, et al., 2020. Caloric Restriction Reprograms the Single-Cell Transcriptional Landscape of Rattus Norvegicus Aging. Cell pp. 1-41. doi:10.1016/j.cell.2020.02.008. McInnes L, Healy J, Melville J, 2020. UMAP: Uniform Manifold Approximation and Projection for Dimension Reduction. arXiv:1802.03426.

Oliver A, Odena A, Raffel CA, Cubuk ED, Goodfellow I, 2018. Realistic Evaluation of Deep Semi-Supervised Learning Algorithms. In Bengio S, Wallach H, Larochelle H, Grauman K, Cesa-Bianchi N, Garnett R (editors), Advances in Neural Information Processing Systems, volume 31, pp. 3235-3246. Curran Associates, Inc.

Platt JC, 1999. Probabilistic Outputs for Support Vector Machines and Comparisons to Regularized Likelihood Methods. In Advances in Large Margin Classifiers, pp. 61-74. MIT Press.

Pliner HA, Shendure J, Trapnell C, 2019. Supervised classification enables rapid annotation of cell atlases. Nature methods pp. 1-8. doi:10.1038/s41592-019-0535-3.

Sohn K, Berthelot D, Li CL, Zhang Z, Carlini N, Cubuk ED, Kurakin A, Zhang H, Raffel C, 2020. FixMatch: Simplifying Semi-Supervised Learning with Consistency and Confidence. arXiv:2001.07685 [cs, stat] ArXiv: 2001.07685 .

Srivastava N, Hinton GE, Krizhevsky A, 2014. Dropout: a simple way to prevent neural networks from overfitting. Journal of Machine Learning Research 15: 1929-1958.

Srivatsan SR, McFaline-Figueroa JL, Ramani V, Saunders L, Cao J, Packer J, Pliner HA, Jackson DL, Daza RM, Christiansen L, et al., 2019. Massively multiplex chemical transcriptomics at single cell resolution. Science (New York, N.Y.) 367: 45-51.

Stuart T, Butler A, Hoffman P, Hafemeister C, Papalexi E, Mauck III WM, Hao Y, Stoeckius M, Smibert P, Satija R, 2019. Comprehensive Integration of Single-Cell Data. Cell pp. 1-37. doi:10.1016/j.cell.2019.05.031.

Sundararajan M, Taly A, Yan Q, 2017. Axiomatic Attribution for Deep Networks. arXiv:1703.01365 [cs] ArXiv: 1703.01365 .

Svensson V, da Veiga Beltrame E, Pachter L, 2020. A curated database reveals trends in single-cell transcriptomics. Database 2020. doi:10.1093/database/baaa073. Baaa073. 
Svensson V, Vento-Tormo R, Teichmann SA, 2018. Exponential scaling of single-cell RNA-seq in the past decade. Nature Protocols 13: 599-604. doi:10.1038/nprot.2017.149.

Tabula Muris Consortium, 2018. Single-cell transcriptomics of 20 mouse organs creates a Tabula Muris. Nature 562: $367-372$.

Tan Y, Cahan P, 2019. SingleCellNet: A Computational Tool to Classify Single Cell RNA-Seq Data Across Platforms and Across Species. Cell Systems pp. 1-31. doi:10.1016/j.cels.2019.06.004.

Tanay A, Regev A, 2017. Scaling single-cell genomics from phenomenology to mechanism. Nature 541: 331-338.

Thulasidasan S, Chennupati G, Bilmes JA, Bhattacharya T, Michalak S, 2019. On Mixup Training: Improved Calibration and Predictive Uncertainty for Deep Neural Networks. In Advances in Neural Information Processing Systems 32, pp. 13888-13899.

Tran HTN, Ang KS, Chevrier M, Zhang X, Lee NYS, Goh M, Chen J, 2020. A benchmark of batch-effect correction methods for single-cell RNA sequencing data. Genome Biology 21: 12. doi:10.1186/s13059-019-1850-9.

Trapnell C, 2015. Defining cell types and states with single-cell genomics. Genome Research 25: 1491-1498.

Verma V, Lamb A, Kannala J, Bengio Y, Lopez-Paz D, 2019. Interpolation Consistency Training for Semi-supervised Learning. In Proceedings of the 28th International Joint Conference on Artificial Intelligence, IJCAI' 19, pp. 36353641. AAAI Press.

Wilson G, Cook DJ, 2020. A Survey of Unsupervised Deep Domain Adaptation. ACM Trans. Intell. Syst. Technol. 11. doi:10.1145/3400066.

Xu C, Lopez R, Mehlman E, Regier J, Jordan MI, Yosef N, 2019. Harmonization and Annotation of Single-cell Transcriptomics data with Deep Generative Models. bioRxiv pp. 1-46. doi:10.1101/532895.

Zeiler MD, 2012. ADADELTA: An Adaptive Learning Rate Method. arXiv:1212.5701 .

Zhang AW, Flanagan COx, Chavez EA, Jamie L P Lim, Ceglia N, McPherson A, Wiens M, Walters P, Chan T, Hewitson B, et al., 2019. Probabilistic cell-type assignment of single-cell RNA-seq for tumor microenvironment profiling. Nature methods pp. 1-16. doi:10.1038/s41592-019-0529-1.

Zhang H, Cisse M, Dauphin YN, Lopez-Paz D, 2018. mixup: Beyond Empirical Risk Minimization. In International Conference on Learning Representations.

Zhong E, Fan W, Yang Q, Verscheure O, Ren J, 2010. Cross Validation Framework to Choose amongst Models and Datasets for Transfer Learning. In Balcázar JL, Bonchi F, Gionis A, Sebag M (editors), Machine Learning and Knowledge Discovery in Databases, Lecture Notes in Computer Science, pp. 547-562. Springer, Berlin, Heidelberg. doi:10.1007/978-3-642-15939-8_35. 


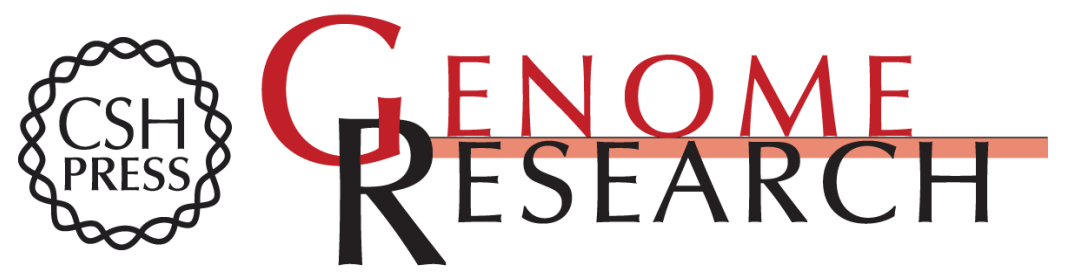

\title{
Semi-supervised adversarial neural networks for single-cell classification
}

\author{
Jacob C. Kimmel and David R Kelley
}

Genome Res. published online February 24, 2021

Access the most recent version at doi:10.1101/gr.268581.120

\section{Supplemental http://genome.cshlp.org/content/suppl/2021/09/09/gr.268581.120.DC1 \\ Material}

Related Content Iterative epigenomic analyses in the same single cell

Hidetaka Ohnuki, David J. Venzon, Alexei Lobanov, et al.

Genome Res. October, 2021 31: 1819-1830 Multimodal single-cell/nucleus RNA

sequencing data analysis uncovers molecular networks between

disease-associated microglia and astrocytes with implications for drug

repurposing in Alzheimer's disease

Jielin Xu, Pengyue Zhang, Yin Huang, et al.

Genome Res. October, 2021 31: 1900-1912 Linear-time cluster ensembles of

large-scale single-cell RNA-seq and multimodal data

Van Hoan Do, Francisca Rojas Ringeling and Stefan Canzar

Genome Res. April , 2021 31:677-688

$\mathbf{P}<\mathbf{P} \quad$ Published online February 24, 2021 in advance of the print journal.

Accepted Peer-reviewed and accepted for publication but not copyedited or typeset; accepted Manuscript manuscript is likely to differ from the final, published version.

Open Access Freely available online through the Genome Research Open Access option.

Creative This manuscript is Open Access. This article, published in Genome Research, is

Commons available under a Creative Commons License (Attribution-NonCommercial 4.0

License International license), as described at

http://creativecommons.org/licenses/by-nc/4.0/.

Email Alerting Receive free email alerts when new articles cite this article - sign up in the box at the Service top right corner of the article or click here.

\section{Affordable, Accurate Sequencing.}

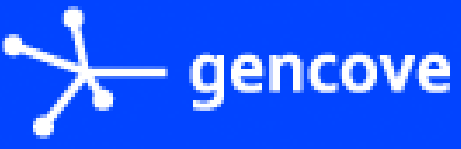

To subscribe to Genome Research go to:

https://genome.cshlp.org/subscriptions 


\section{Affordable, Accurate} Sequencing.

To subscribe to Genome Research go to: https://genome.cshlp.org/subscriptions 